\title{
Dapagliflozin: A Review in Type 2 Diabetes
}

\author{
Sohita Dhillon ${ }^{1}$
}

Published online: 25 June 2019

(c) Springer Nature 2019, corrected publication 2019

\begin{abstract}
Dapagliflozin $\left(\right.$ Forxiga $\left.^{\circledR}\right)$ is a highly potent, reversible and selective sodium-glucose cotransporter-2 inhibitor indicated worldwide for the treatment of type 2 diabetes (T2D). In the EU, oral dapagliflozin once daily is approved for use as monotherapy (in patients who are intolerant of metformin) and as add-on combination therapy (with other glucose-lowering agents, including insulin) for T2D when diet and exercise alone do not provide adequate glycaemic control. In numerous well-designed clinical studies and their extensions, dapagliflozin as monotherapy and combination therapy with other antihyperglycaemic agents provided effective glycaemic control and reduced bodyweight and blood pressure (BP) across a broad spectrum of patients. Dapagliflozin reduced the rate of cardiovascular (CV) death or hospitalization for heart failure (HHF), did not adversely affect major adverse CV events (MACE) and possibly reduced progression of renal disease relative to placebo in patients with established atherosclerotic CV disease (CVD) or multiple risk factors for CVD. Dapagliflozin was generally well tolerated, with a low risk of hypoglycaemia; diabetic ketoacidosis (DKA), although rare, and genital infections were more common with dapagliflozin than placebo. Given its antihyperglycaemic, cardioprotective and possibly renoprotective properties and generally favourable tolerability profile, dapagliflozin provides an important option for the management of a broad patient population, regardless of the history of CVD.
\end{abstract}

\section{Dapagliflozin: clinical considerations in T2D}

Lowers glucose levels independently of insulin action

Provides effective glycaemic control and reduces bodyweight and BP

Reduces rate of CV death or HHF, does not adversely affect MACE and possibly reduces progression of renal disease

Low risk of hypoglycaemia, while genital infections and DKA are more common than with placebo

The manuscript was reviewed by: $G$. Dimitriadis, 2nd Department of Internal Medicine, Research Institute \& Diabetes Center, Athens University Medical School, Attikon University Hospital, Athens, Greece; J. G. Eriksson, Department of General Practice and Primary Health Care, University of Helsinki and Helsinki University Hospital, Helsinki, Finland; N. Papanas, Diabetes Centre, 2nd Department of Internal Medicine, Democritus University of Thrace, Alexandroupolis, Greece; $\boldsymbol{A}$. J. Scheen, Department of Medicine, CHU Liège, University of Liège, Liège, Belgium.

Sohita Dhillon

demail@springer.com

1 Springer Nature, Private Bag 65901, Mairangi Bay, Auckland 0754, New Zealand

\section{Introduction}

Sodium-glucose cotransporter-2 (SGLT2) inhibitors are a relatively new class of antihyperglycaemic agents (AHAs) for the treatment of type 2 diabetes (T2D) [1-3]. These agents reduce reabsorption of glucose in the kidneys and facilitate its excretion in the urine by inhibiting the high-capacity glucose transporter SGLT2 located in the proximal convoluted tubule, thereby lowering glucose levels independently of insulin action $[1,2]$. This unique mechanism of action of SGLT2 inhibitors complements that of other classes of AHAs, allowing for their use as combination therapy with other AHAs, including insulin. Dapagliflozin (Forxiga ${ }^{\circledR}$ ) is one such SGLT2 inhibitor that is approved for the treatment of T2D in various countries worldwide, including the EU and USA. The pharmacological properties and clinical use of dapagliflozin in adults with T2D have been extensively reviewed previously in Drugs [4, 5]. This article, written from an EU perspective, focuses on recent trials, including the large DECLARE-TIMI 58 cardiovascular (CV) outcomes trial in patients with T2D with or without established cardiovascular disease (CVD). Dapagliflozin is also available as fixed-dose dapagliflozin/metformin (Xigduo ${ }^{\circledR}$ ) and dapagliflozin/saxagliptin $\left(\right.$ Qtern $\left.^{\circledR}\right)$ tablets. 


\section{Pharmacological Properties}

Dapagliflozin is a highly potent (inhibitory constant $0.55 \mathrm{nmol} / \mathrm{L}$ ) and reversible SGLT2 inhibitor that is $>1400$ times more selective for SGLT2 than SGLT1, the main transporter responsible for glucose absorption in the gut $[6,7]$. Dapagliflozin increased the amount of glucose excreted in the urine and improved both fasting (FPG) and post-prandial plasma glucose levels in patients with T2D [8]. Urinary glucose excretion (glucuresis) was seen after the first dose of dapagliflozin, was continuous during the $24 \mathrm{~h}$ dosing interval and maintained over the course of therapy [7, 8]. Dapagliflozin-induced glucuresis in patients with T2D was associated with caloric loss and a modest reduction in bodyweight, as well as mild osmotic diuresis and transient natriuresis [7, 9, 10]. The loss in bodyweight with SGLT2 inhibitors is less than that calculated from calorie loss due to glucuresis, which may be because of compensatory mechanisms such as increased energy intake [11]. A modest decrease in blood pressure (BP) was also seen with dapagliflozin, which may be explained by a decrease in circulating volume because of the diuretic/natriuretic properties of the drug [10]. The effects of dapagliflozin on glycaemic parameters, bodyweight and BP in large clinical trials in patients with T2D are summarized in Sect. 3.

Dapagliflozin is rapidly absorbed after oral administration, with peak plasma concentrations usually reached within $2 \mathrm{~h}$ (fasted state) [7]. After a $10 \mathrm{mg}$ dose, the absolute oral bioavailability of dapagliflozin is $78 \%$. The mean steadystate volume of distribution of dapagliflozin is $118 \mathrm{~L}$ and it is $\approx 91 \%$ protein bound. Dapagliflozin pharmacokinetics are not affected by food to a clinically meaningful extent. Dapagliflozin is largely metabolized by UGT1A9 (an enzyme in the liver and kidneys) to its major inactive metabolite 3-O-glucuronide; the major and other metabolites of dapagliflozin do not contribute to its glucose-lowering effects. Dapagliflozin and its metabolites are largely excreted in the urine, with $75 \%$ of a dose recovered in the urine $(<2 \%$ as unchanged parent drug) and $21 \%$ in the faeces $(\approx 15 \%$ as unchanged parent drug). After single-dose dapagliflozin $10 \mathrm{mg}$ in healthy subjects, the mean plasma terminal elimination half-life of dapagliflozin was $12.9 \mathrm{~h}$ [7].

\section{Therapeutic Efficacy of Dapagliflozin}

\subsection{Glycaemic and Other Outcomes}

As reviewed previously in Drugs [4, 5], numerous randomized, double-blind, multicentre, phase 3 trials with dapagliflozin as monotherapy and combination therapy have demonstrated its efficacy in improving glycaemic control and reducing bodyweight and BP in a broad spectrum of patients with T2D, including those with high baseline $\mathrm{HbA}_{1 \mathrm{c}}(\geq 9 \%)$ [12] and the elderly (aged $\geq 65$ years) [13]. Results from more recent trials, including special populations such as patients with chronic kidney disease (CKD) stage 3A [14], hypertension [15, 16] or CVD [17, 18], are summarized in Tables 1 and 2).

In a randomized, double-blind, multinational, phase 3 study in patients inadequately controlled with metformin $(n=182)$, add-on dapagliflozin reduced bodyweight largely by reducing fat mass relative to placebo, with fat mass accounting for approximately two-thirds of the total weight loss [19]. At week 24 , patients receiving add-on dapagliflozin $10 \mathrm{mg}$ once daily had significantly lower total bodyweight (primary endpoint; difference from placebo $-2.1 \mathrm{~kg}$; baseline $\approx 92 \mathrm{~kg}$; $p<0.0001)$, smaller waist circumference $(-1.5 \mathrm{~cm}$; baseline $\approx 105 \mathrm{~cm} p=0.0143$ ) and less fat mass as assessed by dual X-ray absorptiometry (DXA) $(-1.5 \mathrm{~kg}$; baseline $\approx 33 \mathrm{~kg}$; $p=0.0001)$ than those receiving add-on placebo. A rapid decline in bodyweight was seen in the first few weeks of dapagliflozin treatment, with a gradual decline thereafter that had not plateaued at week 24 . This change in bodyweight was reflected in the daily spot urinary glucose level, which showed an initial rapid increase and stable levels thereafter, supporting the DXA findings that the loss in bodyweight and fat mass with dapagliflozin was largely because of caloric loss from glucosuria. However, the initial rapid decline in bodyweight in dapagliflozin recipients may partly be because of fluid loss [19].

Additionally, the proportion of patients with a decrease in bodyweight of $\geq 5 \%$ was significantly higher in patients receiving dapagliflozin than those receiving placebo (31 vs. $4 \% ; \mathrm{p}<0.0001$ ) [19]. Moreover, magnetic resonance imaging in a substudy in 80 patients showed that both visceral and subcutaneous adipose tissues were reduced in dapagliflozin relative to placebo recipients (difference from placebo -258 and $-185 \mathrm{~cm}^{3}$, respectively; both nominal $p<0.05$ ) [19]. The reductions in bodyweight, fat mass and waist circumference with add-on dapagliflozin versus add-on placebo at week 24 were maintained over 102 weeks' therapy [20].

\subsubsection{Patients with Hypertension}

Dapagliflozin $10 \mathrm{mg}$ once daily reduced SBP and improved glycaemic control in two phase 3 studies in patients with inadequately controlled T2D and hypertension despite receiving antihypertensive therapy (angiotensin-converting enzyme inhibitor (ACEi)/angiotensin receptor blocker (ARB) therapy alone [15] or in combination with one other antihypertensive [16]). At week 12, mean SBP and $\mathrm{HbA}_{1 \mathrm{c}}$ were significantly lower with dapagliflozin than placebo in both studies (first and second coprimary endpoints, respectively) (Table 2). A post hoc analysis of one study suggested that SBP was lowered with dapagliflozin to a greater degree in patients receiving a $\beta$ blocker or a calcium-channel blocker as their additional antihypertensive drug than in those receiving a thiazide diuretic [16]. 
Table 1 Efficay of oral dapagliflozin $10 \mathrm{mg}$ once daily as add-on therapy in randomized, double-blind, multicentre, phase 3-4 trials and extensions in patients with T2D

\begin{tabular}{|c|c|c|c|c|c|c|c|}
\hline \multirow{2}{*}{$\begin{array}{l}\text { Study (mean diabetes } \\
\text { duration) }\end{array}$} & \multirow{2}{*}{$\begin{array}{l}\text { Duration } \\
\text { (weeks) }\end{array}$} & \multirow[t]{2}{*}{ Treatment (no. of pts) } & \multicolumn{4}{|c|}{ Adjusted mean ${ }^{\mathrm{a}}$ change from $\mathrm{BL}[\mathrm{BL}]$} & \multirow{2}{*}{$\begin{array}{l}\mathrm{HbA}_{1 \mathrm{c}}<7 \% \\
(\% \text { of pts) }\end{array}$} \\
\hline & & & $\mathrm{HbA}_{1 \mathrm{c}}^{\mathrm{b}}(\%)$ & FPG (mmol/L) & BW (kg) & $\mathrm{SBP}^{\mathrm{c}}(\mathrm{mmHg})$ & \\
\hline \multirow{4}{*}{$\begin{array}{l}\text { Matthaei et al. }[57, \\
62] \text { ( } \approx 9 \text { years })\end{array}$} & \multirow[t]{2}{*}{24} & $\mathrm{DAPA}+\mathrm{MET}+\mathrm{SU}(108)$ & $-0.86 * * *[8.1]$ & $-1.9^{* * *}[9.3]$ & $-2.7 * * *[89]$ & $-4.0^{* \mathrm{c}}[135]$ & $32 * * *$ \\
\hline & & $\mathrm{PL}+\mathrm{MET}+\mathrm{SU}(108)$ & $-0.17[8.2]$ & $-0.04[10.2]$ & $-0.6[90]$ & $-0.3^{\mathrm{c}}[136]$ & 11 \\
\hline & \multirow[t]{2}{*}{$52[57]$} & $\mathrm{DAPA}+\mathrm{MET}+\mathrm{SU}$ & -0.8 & -1.5 & -2.9 & -1.0 & 27 \\
\hline & & $\mathrm{PL}+\mathrm{MET}+\mathrm{SU}$ & -0.2 & +0.6 & -1.0 & +1.1 & 11 \\
\hline \multirow{4}{*}{$\begin{array}{l}\text { Matheiu et al. } \\
\qquad 63] \text { ( } \approx 8 \text { years })\end{array}$} & \multirow[t]{2}{*}{24} & DAPA + SAX + MET (160) & $-0.82 * * *[8.2]$ & $-1.8^{* * *}[9.9]$ & $-1.9 * * *[86]$ & $-1.9 *[\mathrm{NA}]$ & $38 * * *$ \\
\hline & & $\mathrm{PL}+\mathrm{SAX}+\mathrm{MET}(160)$ & $-0.10[8.2]$ & $-0.3[9.8]$ & $-0.4[88]$ & $+2.0[\mathrm{NA}]$ & 12 \\
\hline & \multirow[t]{2}{*}{$52[58]$} & $\mathrm{DAPA}+\mathrm{SAX}+\mathrm{MET}$ & -0.74 & -1.5 & -2.1 & NA & 29 \\
\hline & & $\mathrm{PL}+\mathrm{SAX}+\mathrm{MET}$ & +0.07 & +0.6 & -0.4 & NA & 13 \\
\hline \multirow{3}{*}{$\begin{array}{l}\text { Müller-Wieland et al. } \\
\text { [64] ( } \approx 7 \text { years })\end{array}$} & \multirow[t]{3}{*}{52} & DAPA + SAX + MET (312) & $-1.2^{\dagger \dagger \mathrm{d}}[8.3]$ & $-2.1^{\dagger \dagger}[10.4]$ & $-3.2^{\dagger \dagger}[95]$ & $-6.4^{\dagger \dagger}[139]$ & 40 \\
\hline & & DAPA + MET (311) & $-0.82^{\mathrm{d}}[8.3]$ & $-1.6[10.6]$ & $-3.5^{\dagger \dagger}[98]$ & $-5.6^{\dagger \dagger}[138]$ & $20^{\dagger \dagger}$ \\
\hline & & GLIM + MET (309) & $-0.99^{\mathrm{d}}[8.3]$ & $-1.5[10.4]$ & $+1.8[98]$ & $-1.6[139]$ & 34 \\
\hline \multirow{4}{*}{$\begin{array}{l}\text { Handelsman et al. } \\
{[65](\approx 8 \text { years })}\end{array}$} & \multirow[t]{2}{*}{26} & DAPA + SAX + MET (232) & $-1.41^{\dagger \dagger}[8.8]$ & $-1.8^{\dagger \dagger \dagger}[9.5]$ & $-1.9^{\dagger \dagger}$ & NA & $37^{\dagger}$ \\
\hline & & SIT + MET (229) & $-1.07[8.9]$ & $-0.6[9.7]$ & -0.5 & NA & 25 \\
\hline & \multirow[t]{2}{*}{52} & $\mathrm{DAPA}+\mathrm{SAX}+\mathrm{MET}$ & -1.29 & -1.4 & -2.3 & $-2.6[130]$ & 33 \\
\hline & & $\mathrm{SIT}+\mathrm{MET}$ & -0.81 & -0.2 & -0.8 & $+2.5[129]$ & 20 \\
\hline \multirow{6}{*}{$\begin{array}{l}\text { Frias et al. }[61] \\
\text { (DURATION-8) } \\
\quad(\approx 7 \text { years })\end{array}$} & \multirow[t]{3}{*}{28} & DAPA + EXN + MET (228) & $-2.0^{\S \dagger}[9.3]$ & $-3.66^{\S \dagger \dagger}[10.8]$ & $-3.6^{\S+\dagger}[92]$ & $-4.3^{\dagger}[131]$ & $45^{\S \dagger \dagger}$ \\
\hline & & $\mathrm{DAPA}+\mathrm{PL}+\mathrm{MET}(227)$ & $-1.4[9.3]$ & $-2.73[10.5]$ & $-2.2[91]$ & $-1.8^{\dagger}[130]$ & 19 \\
\hline & & $\mathrm{PL}+\mathrm{EXN}+\mathrm{MET}(230)$ & $-1.6[9.3]$ & $-2.54[10.5]$ & $-1.6[89]$ & $-1.2[129]$ & 27 \\
\hline & \multirow[t]{3}{*}{$104[60]^{\mathrm{e}}$} & $\mathrm{DAPA}+\mathrm{EXN}+\mathrm{MET}$ & $-1.70^{\S \dagger}$ & $-2.70^{\S+\dagger}$ & $-2.5^{\dagger}$ & $-3.1^{\dagger}$ & NA \\
\hline & & $\mathrm{DAPA}+\mathrm{PL}+\mathrm{MET}$ & -1.06 & -1.20 & -3.0 & -1.1 & NA \\
\hline & & $\mathrm{PL}+\mathrm{EXN}+\mathrm{MET}$ & -1.29 & -1.70 & -0.8 & -0.1 & NA \\
\hline \multirow{4}{*}{$\begin{array}{l}\text { Vilsbøll et al. [66] } \\
\text { (NA) }\end{array}$} & \multirow[t]{2}{*}{24} & $\mathrm{DAPA}+\mathrm{SAX}+\mathrm{MET} \pm \mathrm{SU}(324)$ & $-1.7^{\mathrm{d}}[9.0]$ & & $-1.5^{\dagger \dagger}$ & NA & $21^{\dagger \mathrm{f}}$ \\
\hline & & $\mathrm{INS}+\mathrm{MET} \pm \mathrm{SU}(319)$ & $-1.5^{\mathrm{d}}[9.0]$ & & +2.1 & NA & $13^{\mathrm{f}}$ \\
\hline & \multirow[t]{2}{*}{$52[59]^{\mathrm{e}}$} & $\mathrm{DAPA}+\mathrm{SAX}+\mathrm{MET} \pm \mathrm{SU}$ & $-1.51^{\dagger}$ & & $-1.83^{\dagger \dagger}$ & NA & $15^{\dagger \dagger f}$ \\
\hline & & $\mathrm{INS}+\mathrm{ME} \pm \mathrm{SU}$ & -1.26 & & +2.75 & NA & $7^{f}$ \\
\hline
\end{tabular}

No statistical comparisons available for two extension studies $[57,58]$ and nominal $p$ values reported for two others $[59,60]$

$B L$ baseline, $B W$ bodyweight, $D A P A$ oral dapagliflozin $10 \mathrm{mg} /$ day, $E X N$ subcutaneous exenatide-extended release $2 \mathrm{mg}$ once weekly, $F P G$ fasting plasma glucose, GLIM oral glimepiride $4 \mathrm{mg} /$ day, $I N S$ titrated insulin glargine (FPG goal $\leq 5.5 \mathrm{mmol} / \mathrm{L}$ ), $H b A_{l c}$ glycated haemoglobin, $M E T$ oral metformin $\geq 1500 \mathrm{mg} /$ day, $N A$ not available, $P L$ placebo, pts patients, $S I T$ oral sitagliptin $100 \mathrm{mg} /$ day, $S A X$ oral saxagliptin $5 \mathrm{mg} /$ day, $S B P$ systolic blood pressure, $S U$ oral sulfonylurea $\geq 50 \%$ of maximum dose

${ }^{*} p<0.05,{ }^{* *} p \leq 0.001,{ }^{* * *} p<0.0001$ vs. PL; ${ }^{\dagger} p<0.05,{ }^{\dagger \dagger} p \leq 0.001,{ }^{\dagger \dagger} p<0.0001$ vs. active comparator; ${ }^{\S} p<0.001$ vs. DAPA

${ }^{a}$ Values are least-squares mean in two studies $[60,61]$

${ }^{\mathrm{b}}$ Primary endpoint for the main study

${ }^{\mathrm{c}} \mathrm{SBP}$ assessed at week 8 in one study [62]

${ }^{\mathrm{d}}$ Noninferiority between treatment groups was demonstrated

eAbstract presentation

${ }^{\mathrm{f}}$ Proportion of patients achieving target $\mathrm{HbA} 1 \mathrm{c}<7 \%$ without hypoglycaemia

\subsubsection{Patients with Cardiovascular Disease}

Dapagliflozin $10 \mathrm{mg}$ once daily improved glycaemic control and reduced bodyweight and SBP when added to usual background therapy in two phase 3 studies in patients with inadequately controlled T2D ( $\mathrm{HbA}_{1 \mathrm{c}} 7-10.5 \%$ across the studies) and pre-existing CVD and hypertension [17, 18]. At week 24, in both studies, $\mathrm{HbA}_{1 \mathrm{c}}$ was lowered to a significantly greater extent with add-on dapagliflozin than add-on placebo, and a significantly greater proportion of patients in the dapagliflozin group had a 3-item response than in the placebo group (coprimary endpoints; Table 2) [17, 18]. Treatment benefits with dapagliflozin were maintained at week 52 in the extension studies $[17,18]$. A significantly $(p<0.005)$ greater proportion of patients in the dapagliflozin than placebo groups in both studies achieved a target $\mathrm{HbA}_{1 \mathrm{c}}$ of $<7 \%$ at week 24 (19 vs. $13 \%$ [17]; 16 vs. 8\% [18]), with the between-group differences maintained at week 52 (19 vs. $10 \%$ [17]; 15 vs. 5\% [18]). Efficacy of dapagliflozin was maintained during longer-term treatment in a second extension of 52 weeks (total 104 weeks' therapy, according to a post hoc pooled analysis of the two studies [21].

In a pooled analysis of five, phase 2-3 clinical trials of $\leq 52$ weeks' duration, patients with T2D and a history of HF had clinically meaningful reductions from baseline in $\mathrm{HbA}_{1 \mathrm{c}}$ 
Table 2 Efficay of oral dapagliflozin $\mathbf{1 0} \mathbf{~ m g}$ once daily as add-on to existing antidiabetic therapy in randomized, double-blind, multicentre, phase 3 trials and extensions in patients with T2D and high-risk of cardiovascular complications

\begin{tabular}{|c|c|c|c|c|c|c|c|}
\hline \multirow[t]{2}{*}{ Study } & \multirow{2}{*}{$\begin{array}{l}\text { Duration } \\
\text { (weeks) }\end{array}$} & \multirow{2}{*}{$\begin{array}{l}\text { Treatment } \\
\text { (no. of pts) }\end{array}$} & \multicolumn{4}{|c|}{ Adjusted mean change from BL [BL] } & \multirow{2}{*}{$\begin{array}{l}\text { 3-item response } \\
\text { (\% of pts) }\end{array}$} \\
\hline & & & $\mathrm{HbA}_{1 \mathrm{c}}(\%)$ & $\mathrm{FPG}(\mathrm{mmol} / \mathrm{L})$ & Bodyweight (kg) & $\mathrm{SBP}(\mathrm{mmHg})$ & \\
\hline \multicolumn{8}{|c|}{ In pts with hypertension on ACEi or ARB therapy } \\
\hline \multirow[t]{2}{*}{ Weber et al. [15] } & 12 & DAPA (302) & $-0.6^{* * * b, c}[8.1]$ & $-0.7^{\mathrm{c}}[8.8]$ & $-1.0^{\mathrm{c}}[86]$ & $-10.4^{* * \mathrm{~b}, \mathrm{c}}[150]$ & \\
\hline & & PL (311) & $-0.1^{\mathrm{b}, \mathrm{c}}[8.0]$ & $+0.4^{\mathrm{c}}[8.9]$ & $-0.3^{\mathrm{c}}[84]$ & $-7.3^{\mathrm{b}, \mathrm{c}}[150]$ & \\
\hline \multicolumn{8}{|c|}{ In pts with hypertension on combination antihypertensive therapy } \\
\hline \multirow[t]{2}{*}{ Weber et al. [16] } & 12 & DAPA (225) & $-0.63^{* * * b, c}[8.1]$ & $-1.0^{\mathrm{c}}[9.0]$ & $-1.44^{\mathrm{c}}[88]$ & $-11.9^{* * \mathrm{~b}, \mathrm{c}}[151]$ & \\
\hline & & PL (224) & $-0.02^{\mathrm{b}, \mathrm{c}}[8.0]$ & $+0.2^{\mathrm{c}}[8.9]$ & $-0.59^{\mathrm{c}}[90]$ & $-7.6^{\mathrm{b}, \mathrm{c}}[151]$ & \\
\hline \multicolumn{8}{|l|}{ In pts with CVD } \\
\hline \multirow[t]{4}{*}{ Leiter et al. [17] } & 24 & DAPA (480) & $-0.3 * * \mathrm{~b}[8.0]$ & $-0.8 * *[9.0]$ & $-2.5^{* *}[95]$ & $-1.9^{* *}[135]$ & $10 * * \mathrm{~b}$ \\
\hline & & PL (482) & $+0.1^{\mathrm{b}}[8.1]$ & $+0.6[9.2]$ & $-0.6[93]$ & $+0.9[135]$ & $1.9^{\mathrm{b}}$ \\
\hline & 52 & DAPA & -0.5 & -0.9 & -3.2 & -3.6 & 10.6 \\
\hline & & $\mathrm{PL}$ & 0.0 & +0.2 & -1.1 & -0.9 & 3.1 \\
\hline \multirow[t]{4}{*}{ Cefalu et al. [18] } & 24 & DAPA (455) & $-0.38 * * * \mathrm{~b}[8.2]$ & $-0.57 *[8.9]$ & $-2.6 * * *[93]$ & $-2.99^{*}[133]$ & $12 * * * b$ \\
\hline & & PL (459) & $+0.08^{\mathrm{b}}[8.1]$ & $+0.35[8.8]$ & $-0.3[94]$ & $-1.0[133]$ & $1^{\mathrm{b}}$ \\
\hline & 52 & DAPA & -0.44 & -0.96 & -2.9 & -3.40 & 7 \\
\hline & & $\mathrm{PL}$ & +0.22 & -0.01 & -0.3 & +0.18 & 0.7 \\
\hline
\end{tabular}

No statistical comparisons are available for extension studies

$A C E i$ angiotensin-converting enzyme inhibitor, $A R B$ angiotensin receptor blocker, $B L$ baseline, $C V D$ cardiovascular disease, $D A P A$ dapagliflozin, $F P G$ fasting plasma glucose, $H b A_{l c}$ glycated haemoglobin, $P L$ placebo, pts patients, $S B P$ systolic blood pressure

$* p<0.05, * * p \leq 0.001, * * * p<0.0001$ vs. PL

${ }^{a}$ Defined as the proportion of pts achieving combined reduction in HbA1c of $\geq 5.5 \%$, bodyweight of $\geq 3 \%$ and SBP of $\geq 3 \mathrm{mmHg}$

${ }^{\mathrm{b}}$ Coprimary endpoint

${ }^{\mathrm{c}}$ Hierarchical testing was used for the coprimary (mean change in seated SBP followed by mean change in HbA1c) and secondary endpoints

(placebo-adjusted mean change $-0.55 \%$; baseline $8.2 \%$ ), bodyweight $(-2.7 \mathrm{~kg}$; baseline $\approx 97 \mathrm{~kg})$ and SBP $(-2.1 \mathrm{mmHg}$; baseline $\approx 134 \mathrm{mmHg}$ ) with dapagliflozin $10 \mathrm{mg}$ monotherapy or add-on therapy to other AHA regimens $(n=171)$ relative to placebo/active comparator $(n=149)$ [22].

\subsubsection{Patients with Renal Impairment}

A post hoc analysis of a phase $2 / 3$ study suggested treatment benefit with dapagliflozin in patients with CKD stage $3 \mathrm{~A}$ (eGFR $\geq 45$ and $<60 \mathrm{~mL} / \mathrm{min} 1.73 \mathrm{~m}^{2}$ ) [14], with findings supported by the randomized, double-blind, multinational, phase 3 DERIVE study [23]. In DERIVE, patients with inadequately controlled T2D $\left(\mathrm{HbA}_{1 \mathrm{c}} 7-11 \%\right)$, a BMI of $18-45 \mathrm{~kg} /$ $\mathrm{m}^{2}$ who were receiving other AHA regimens and who had CKD stage $3 \mathrm{~A}$ were randomized to receive 24 weeks of dapagliflozin $10 \mathrm{mg}$ once daily $(n=159)$ or placebo $(n=161)$. At week 24 , dapagliflozin significantly $\left(p=0.05\right.$ ) lowered $\mathrm{HbA}_{1 \mathrm{c}}$ (primary endpoint; placebo-adjusted mean change -0.34 ; baseline $\approx$ $8.2 \%$ ), FPG (- $0.9 \mathrm{mmol} / \mathrm{L}$; baseline $\approx 10 \mathrm{mmol} / \mathrm{L})$, bodyweight $(-1.3 \mathrm{~kg}$; baseline $\approx 90 \mathrm{~kg})$ and $\mathrm{SBP}(-3.1 \mathrm{mmHg}$; baseline $\approx 135 \mathrm{mmHg}$ ) relative to placebo [23]. A randomized, double-blind, multinational, phase 3 study (CompoSIT-R) in patients inadequately controlled with metformin \pm sulfonylurea $\left(\mathrm{HbA}_{1 \mathrm{c}} 7-9.5 \%\right)$ and who had mild renal impairment (eGFR $\geq 60$ to $<90 \mathrm{~mL} / \mathrm{min} / 1.73 \mathrm{~m}^{2}$ ) showed that the addition of sitagliptin $100 \mathrm{mg}$ once daily $(n=307)$ was noninferior (primary hypothesis) and superior to that of dapagliflozin 10 once daily $(n=306)$ in improving glycaemic control $\left[\mathrm{HbA}_{1 \mathrm{c}}\right.$ least squares (LS) mean change from baseline -0.51 vs. $-0.36 \%$; baseline $\approx 7.8 \% p=0.006$ ] [24].

\subsection{Cardiovascular and Renal Outcomes}

The effects of dapagliflozin on $\mathrm{CV}$ and renal outcomes were assessed in the randomized, double-blind phase 3 DECLARE-TIMI 58 trial in patients aged $\geq 40$ years with T2D $\left(\mathrm{HbA}_{1 \mathrm{c}} \geq 6.5\right.$ to $\left.<12 \%\right)$ and established atherosclerotic CVD (ASCVD) or multiple risk factors for ASCVD [25]. Patients were also required to have creatinine clearance $\left(\mathrm{CL}_{\mathrm{CR}}\right)$ $\geq 60 \mathrm{~mL} / \mathrm{min}$. Patients with multiple risk factors were men aged $\geq 55$ years or women aged $\geq 60$ years with one or more of traditional risk factors, such as hypertension, dyslipidaemia [i.e. low-density lipoprotein level $>130 \mathrm{mg} / \mathrm{dL}(3.36 \mathrm{mmol} / \mathrm{L})$ or use of lipid-lowering therapies] or use of tobacco [25].

The study was originally designed to assess the effect of dapagliflozin on the primary safety outcome of major adverse CV events (MACE) [25, 26]. However, based on the findings of the EMPA-REG OUTCOME trial of empagliflozin [27] that emerged during the conduct of DECLARE-TIMI 58 , its study design was modified to include dual primary efficacy outcomes of MACE and the composite of CV death 
and hospitalization for heart failure (CV death/HHF) [25, 26]. The two prespecified secondary endpoints were renal composite outcome (see Table 3 for definition) and death from any cause $[25,26]$. At baseline, patients $(n=17,160$ randomized) had a mean age of 64 years and $41 \%$ had established ASCVD, including coronary artery disease (33\% of patients) and heart failure (HF; 10\%) [25]. The mean duration of diabetes was $\approx 11$ years, mean $\mathrm{HbA}_{1 \mathrm{c}}$ was $8.3 \%$ and the mean estimated glomerular filtration rate (eGFR) was $85 \mathrm{~mL} / \mathrm{min} / 1.73 \mathrm{~m}^{2}$ (45 and $7 \%$ of patients had an eGFR of $60-90$ and $<60 \mathrm{~mL} / \mathrm{min} / 1.73 \mathrm{~m}^{2}$, respectively]. Patients were randomized to receive dapagliflozin $10 \mathrm{mg}$ once daily or placebo in addition to other AHAs; use of other AHAs was at the discretion of the treating physician. The median follow-up duration was 4.2 years (69,547 patient-years) [25].

Dapagliflozin significantly lowered the rate of CV death/ HHF versus placebo, but there was no significant betweengroup difference in the rate of MACE (dual efficacy endpoints assessed after confirming the noninferiority of dapagliflozin and placebo for the primary safety outcome of MACE) (Table 3) [25]. As MACE was not lowered to a significant extent with dapagliflozin versus placebo, analyses of secondary and other endpoints (Table 3 ) conducted hierarchically were only hypothesis generating. The lower rate of the composite endpoint of $\mathrm{CV}$ death/HHF in dapagliflozin recipients was attributed to the lower rate of hospitalization for heart failure (HHF) with dapagliflozin than placebo; the rate of $\mathrm{CV}$ death was generally similar between the two groups (Table 3). Sensitivity analyses of the primary efficacy endpoints supported the findings of the primary analysis of the outcomes [25].

In terms of secondary and other endpoints, results suggest that dapagliflozin reduces the likelihood of progression of renal disease, as indicated by lower incidences of the renal composite and additional renal composite outcomes in dapagliflozin than placebo recipients (Table 3) [25]. For the individual components of the renal outcomes, dapagliflozin relative to placebo was associated with significant reduction in sustained decline in eGFR by $\geq 40 \%$ to $<60 \mathrm{~mL} / \mathrm{min}$ per $1.73 \mathrm{~m}^{2}$ [hazard ratio (HR) 0.54; 95\% CI 0.43-0.67; $p<0.0001$ ), end-stage renal disease (ESRD; HR 0.31; 95\% CI 0.13-0.79; $p=0.013$ ), and renal death or ESRD (HR 0.41; 95\% CI 0.20-0.82; $p=0.012$ ) [28]. The mean decrease from baseline in eGFR was significantly $(p<0.0001)$ greater with dapagliflozin than placebo at 6 months, but had equalized with placebo by 2 years, and was significantly $(p<0.0001)$ less than that with placebo by 3 and 4 years after randomization [28].

The rates of death from any cause, myocardial infarction (MI), ischaemic stroke and death from non-CV causes were generally similar between the two groups (Table 3 ). CV risk factors were improved with dapagliflozin, including the level of $\mathrm{HbA}_{1 \mathrm{c}}$, which was lower in dapagliflozin than placebo recipients throughout the trial (average LS mean absolute difference between groups $0.42 \%$; 95\% CI 0.40-0.45) [25].
Bodyweight (LS mean difference between groups $1.8 \mathrm{~kg} ; 95 \%$ CI 1.7-2.0), SBP (2.7 mmHg; 95\% CI 2.4-3.0) and diastolic BP (DBP; $0.7 \mathrm{mmHg}$; 95\% CI 0.6-0.9) were also lower with dapagliflozin than placebo during the trial [25].

\subsubsection{Subgroup Analyses}

In subgroups analyses, the benefit of dapagliflozin in preventing CV death/HHF was consistent across subgroups, regardless of the history of CVD [patients with ASCVD (HR 0.83; 95\% CI 0.71-0.98) or multiple risk factors (HR $0.84 ; 95 \%$ CI $0.67-1.04)$ ], history of HF (yes or no) or baseline eGFR $(\geq 90$, 60 to $<90$, or $<60 \mathrm{~mL} / \mathrm{min} / 1.73 \mathrm{~m}^{2}$ ) (all $p$ interactions nonsignificant) [25]. The rate of MACE did not differ significantly between dapagliflozin and placebo recipients in any subgroup (all $p$ interactions nonsignificant), including patients with ASCVD (HR 0.90; 95\% CI 0.79-1.02) or multiple risk factors (HR 1.01; 95\% CI 0.86-1.20). Treatment benefit with dapagliflozin relative to placebo in terms of lower rate of progression of renal disease was seen regardless of the history of CVD, $\mathrm{HF}$ or CKD at baseline (all $p$ interactions nonsignificant) [25].

A prespecified subgroup analysis suggested robust clinical benefit with dapagliflozin in the high-risk subgroup of patients with T2D and prior MI (median duration from last event 5.4 years) both in terms of MACE and the composite of CV death/HHF [29]. Dapagliflozin significantly reduced MACE by $16 \%$ (HR $0.84 ; 95 \%$ CI $0.72-0.99 ; p=0.04$ ) in patients with prior MI $(n=3584)$, but not in those with no prior MI ( $n$ $=6771 ;$ HR $1.00 ; 95 \%$ CI $0.88-1.13)$ or no prior MI but with established ASCVD $(n=3390 ;$ HR 0.98; 95\% CI 0.81-1.19). The lower rate of MACE in patients with prior MI was largely because of a lower rate of recurrent MI (HR 0.78; 95\% CI 0.63-0.95). The absolute risk reduction (ARR) for MACE in patients with prior MI was $2.6 \%$ and the number needed to treat (NNT) over 4 years was 39. The treatment benefit with dapagliflozin in terms of MACE appeared to be greater the closer the patients were to the last acute event ( $p$ interaction trend 0.007), with the greatest benefit in patients with a recent MI (> 12 to 24 months). In contrast to MACE, a treatment benefit $(\mathrm{HR}<1)$ for $\mathrm{CV}$ death/HHF was seen with dapaglilozin relative to placebo in all subgroups, i.e. patients with prior MI (HR $0.81 ; 95 \%$ CI $0.65-1.00, p=0.046)$, those with no prior MI (HR 0.85; 95\% CI 0.72-1.00) and patients with no prior MI but with ASCVD (HR 0.87; 95\% CI 0.68-1.12); the ARR in the three patient groups was $1.9 \%$ (NNT over 4 years was 53$), 1.0 \%$ and $0.5 \%$ respectively [29].

Another prespecified subgroup analysis assessed the efficacy of dapagliflozin in patients with T2D and HF with reduced ejection fraction $[\mathrm{HFrEF}$; ejection fraction $(\mathrm{EF})$ $<45 \% ; n=671$ ] and in those without HFrEF [comprising patients with $\mathrm{HF}$ without known reduced $\mathrm{EF}(n=1316)$ and patients without HF $(n=15,173)$ ] [30]. Dapagliflozin reduced $\mathrm{CV}$ death/HHF to a greater extent in patients with $\mathrm{HFrEF}$ (HR 
Table 3 Efficacy of oral dapagliflozin $10 \mathrm{mg}$ once daily in in the DECLARE-TIMI 58 cardiovascular outcomes trial [25]

Outcomes

Event rate (\%) [per 1000 patient-years]

\begin{tabular}{|c|c|c|c|}
\hline & & & \\
\hline & DAPA $(n=8582)$ & $\operatorname{PL}(n=8578)$ & HR vs. PL (95\% CI) \\
\hline \multicolumn{4}{|l|}{ Primary efficacy outcomes } \\
\hline $\mathrm{CV}$ death or $\mathrm{HHF}^{\mathrm{a}}$ & $4.9[12.2]$ & $5.8[14.7]$ & $0.83(0.73-0.95)^{* \mathrm{~b}}$ \\
\hline $\mathrm{MACE}^{\mathrm{a}}$ & $8.8[22.6]$ & $9.4[24.2]$ & $0.93(0.84-1.03)^{\mathrm{b}}$ \\
\hline \multicolumn{4}{|l|}{ Secondary outcomes ${ }^{c}$} \\
\hline Renal composite ${ }^{\mathrm{a}, \mathrm{d}}$ & $4.3[10.8]$ & $5.6[14.1]$ & $0.76(0.67-0.87)$ \\
\hline Death from any cause ${ }^{a}$ & $6.2[15.1]$ & $6.6[16.4]$ & $0.93(0.82-1.04)$ \\
\hline \multicolumn{4}{|l|}{ Other outcomes $^{c}$} \\
\hline Additional renal composite ${ }^{\mathrm{a}, \mathrm{e}}$ & $1.5[3.7]$ & $2.8[7.0]$ & $0.53(0.43-0.66)$ \\
\hline HHF & $2.5[6.2]$ & $3.3[8.5]$ & $0.73(0.61-0.88)$ \\
\hline MI & $4.6[11.7]$ & $5.1[13.2]$ & $0.89(0.77-1.01)$ \\
\hline Ischaemic stroke & $2.7[6.9]$ & $2.7[6.8]$ & $1.01(0.84-1.21)$ \\
\hline Death from CV cause & $2.9[7.0]$ & $2.9[7.1]$ & $0.98(0.82-1.17)$ \\
\hline Death from non CV cause & $2.5[6.0]$ & $2.8[6.8]$ & $0.88(0.73-1.06)$ \\
\hline
\end{tabular}

$C V$ cardiovascular, DAPA dapagliflozin, $e G F R$ estimated glomerular filtration rate, $H H F$ hospitalization for heart failure, $H R$ hazard ratio, $M A C E$ major adverse cardiovascular event (CV death, MI or ischemic stroke), $M I$ myocardial infarction, $P L$ placebo

$* p=0.005$

${ }^{\text {a }}$ Prespecified outcomes

${ }^{\mathrm{b}}$ After demonstrating the noninferiority of DAPA vs. PL $(\mathrm{p}<0.001)$ for the primary safety outcome of MACE, superiority (two-sided $\alpha$ level of 0.023) of DAPA over PL was demonstrated for the endpoint of CV death or HHF, but not for MACE

${ }^{\mathrm{c}}$ Statistical analyses are hypothesis generating because of hierarchical testing

${ }^{\mathrm{d}}$ Defined as $\geq 40 \%$ decrease in eGFR to $<60 \mathrm{ml} / \mathrm{min} / 1.73 \mathrm{~m}^{2}$, end-stage renal disease, or death from renal or CV cause

${ }^{e}$ Defined as $\geq 40 \%$ decrease in eGFR to $<60 \mathrm{ml} / \mathrm{min} / 1.73 \mathrm{~m}^{2}$, end-stage renal disease, or death from renal cause

vs. placebo 0.62 ; $95 \%$ CI $0.45-0.86)$ than in those without HFrEF (HR vs. placebo $0.88,95 \%$ CI $0.76-1.02$ ) ( $p$ interaction 0.046 ), with the difference largely because of a reduction in CV death in patients with HFrEF (HR vs. placebo 0.55; 95\% CI 0.34-0.90, $p=0.02$ ) compared with patients without known HFrEF (HR vs. placebo 1.08; 95\% CI 0.89-1.31). Allcause death was also significantly reduced with dapagliflozin relative to placebo in patients with HFrEF (HR 0.59; 95\% CI $0.40-0.88, p=0.01$ ), but not in those without known HFrEF (HR 0.97; 95\% CI 0.86-1.10) ( $p$ interaction 0.016). The NNT over 4 years for $\mathrm{CV}$ death/HHF, CV death and all-cause death in patients with HFrEF was 11, 19 and 16, respectively. In contrast, HHF was reduced with dapagliflozin regardless of baseline EF, with similar reductions in patients with $\mathrm{HFrEF}$ (HR 0.64; 95\% CI 0.43-0.95) and without known HFrEF (HR $0.76 ; 95 \%$ CI $0.62-0.92$ ) ( $p$ interaction 0.45 ) [30].

\subsection{Real-World Studies}

Several large real-world studies $(n \geq 1900)$ supported the efficacy of dapagliflozin in patients with T2D [31-36]. Dapagliflozin (in addition to other AHAs) for 12 weeks to > 12-24 months numerically or significantly improved efficacy outcomes, including $\mathrm{HbA}_{1 \mathrm{c}}$, bodyweight and SBP in database studies in the UK [31] and USA [32], a Korean postmarketing study [33] and a prospective, multicentre, observational study in India [34]. During 3-6 months' follow-up in a study based on clinical data from a Canadian registry for patients with T2D (for $>6$ months) who received dapagliflozin $(n=1850)$, a dipeptidyl peptidase-4 inhibitor (DPP-4i; $n=1341$ ) or a sulfonylurea ( $n=579)$, significantly more patients receiving dapagliflozin than those receiving a DPP- $4 \mathrm{i}$ or a sulfonylurea had $\mathrm{HbA}_{1 \mathrm{c}}$ reduction of $\geq 0.5 \%$, any weight loss and SBP reduction of $\geq$ $5.0 \mathrm{mmHg}$ (composite primary endpoint; 26 vs. 21 and 15\%; $p<0.05$ ) [35]. Another study, the DARWIN-T2D Italian, multicentre, retrospective study, found that treatment with dapagliflozin $(n=830)$ or a glucagon-like peptide-1 receptor agonist (GLP-1RA; $n=811$ ) significantly $(p<0.05)$ reduced $\mathrm{HbA}_{1 \mathrm{c}}$, bodyweight and SBP [36]. In comparison, treatment with a DPP-4i $(n=2999)$ significantly $(p<0.05)$ reduced two of the three parameters, i.e. $\mathrm{HbA}_{1 \mathrm{c}}$ and bodyweight, and gliclazide (a sulfonylurea; $n=2111)$ significantly $(p<0.05)$ lowered only $\mathrm{HbA}_{1 \mathrm{c}}$. While the reduction from baseline in $\mathrm{HbA}_{1 \mathrm{c}}$ was generally similar in all four treatment groups (change from baseline $-0.7,-0.6,-0.6$ and $-0.6 \%$ ), treatment with dapagliflozin or a GLP-1RA was associated with numerically greater improvements in bodyweight (change from baseline -2.7 and -2.4 vs. -0.5 and $-0.1 \mathrm{~kg})$ and $\mathrm{SBP}(-3.0$ and -1.4 vs. -0.7 and $+0.1 \mathrm{mmHg}$ ) than treatment with a DPP-4i or gliclazide [36].

The efficacy of dapagliflozin in preventing $\mathrm{CV}$ events in DECLARE-TIMI 58 was supported by real-world experience in the CVD-REAL Nordic study, based on data from nationwide 
registries in Denmark, Norway and Sweden for patients with T2D who were prescribed AHAs during 2012-2015 [37]. Dapagliflozin ( $n=10,227$ patients) significantly lowered the risk of MACE (non-fatal MI, non-fatal stroke or CV mortality; HR 0.79; 95\% CI 0.67-0.94), HHF (HR 0.62; 95\% CI $0.50-0.77$ ) and all-cause death (HR $0.59 ; 95 \%$ CI $0.49-0.72$ ) versus DPP-4is $(n=30,681)$ after a mean follow-up of 0.95 years [37].

\section{Tolerability of Dapagliflozin}

Dapagliflozin $10 \mathrm{mg}$ once daily as monotherapy and as add-on therapy to other AHAs was generally well tolerated in patients with T2D in pooled data from 13-30 placebo-/active comparator-controlled phase $2 \mathrm{~b} / 3$ clinical trials of 24 to $\leq 208$ weeks' duration [38]. In a pooled analysis of 13, placebo-controlled trials of 12-24 weeks' duration, treatment-emergent adverse events (AEs) were reported in 60\% (1416/2360) of dapagliflozin and $56 \%$ (1279/2295) of placebo recipients, with $4 \%$ of patients in each group discontinuing therapy because of these events. The most common (incidence $\geq 3 \%$ ) treatment-emergent AEs with dapagliflozin were nasopharyngitis (5 vs. $6 \%$ with placebo), diarrhoea (3 vs. $4 \%$ ), headache (3 vs. $4 \%$ ), upper respiratory tract infections (3 vs. $4 \%$ ), urinary tract infection (UTIs; 4 vs. 3\%) and back pain (4 vs. 2\%). Serious adverse events (SAEs) occurred in $5 \%$ of patients in each group and resulted in treatment discontinuation in $0.7 \%$ of dapagliflozin and $1 \%$ of placebo recipients; deaths were infrequent in both groups (0.3 and $0.2 \%$, respectively) [38]. In the DECLARETIMI 58 trial, significantly more dapagliflozin than placebo recipients discontinued the trial regimen because of AEs (8 vs. $7 \% ; p=0.01)$, but significantly fewer dapagliflozin than placebo recipients had SAEs (34 vs. 36\%; p < 0.001) (safety population $n=8574$ and 8569 in the respective groups) [25]. The most common (incidence $>2 \%$ ) SAEs were unstable angina (2.8 vs. $2.8 \%)$ and acute MI (2.7 vs. $2.3 \%)$ [25].

\subsection{Adverse Events of Special Interest}

Hypoglycaemia occurred in $14 \%$ of dapagliflozin and $12 \%$ of placebo recipients in the 13-study pooled analysis [38]. Three major hypoglycaemic events were reported in the dapagliflozin group and two in the placebo group, with most events occurring in patients receiving insulin as background therapy; one event in a patient receiving dapagliflozin plus insulin and metformin resulted in discontinuation of therapy [38]. In DECLARE-TIMI 58, major hypoglycaemic events occurred in significantly fewer dapagliflozin than placebo recipients (0.7 vs. $1.0 \% ; p=0.02)$ [25].

Genital infections were more frequent with dapagliflozin than placebo in the 13 -study pooled analysis (5.5\% vs. $0.6 \%)$, occurring at least twice as often in women than in men in both treatment groups [38]. All genital infections were of mild or moderate severity, with only $0.2 \%$ of patients in the dapagliflozin group and none in the placebo group requiring treatment discontinuation [38]. UTIs were reported in 5\% of dapagliflozin and $4 \%$ of placebo recipients in this analysis, occurring almost five times more frequently in women than in men, regardless of the treatment group. Most UTIs were of mild or moderate severity, had flora consistent with those in patients with T2D, were not kidney infections and did not require discontinuation of therapy (UTI-related discontinuation rate $\leq 0.2 \%$ in both groups). Most patients with genital infections or UTIs in both treatment groups responded to initial antimicrobial therapy and did not require additional treatment [38]. Results from DECLARE-TIMI 58 supported the findings of the pooled analysis, with genital infections being more common with dapagliflozin than placebo $(0.9$ vs. $0.1 \%$; $p<0.001)$, while there was no significant between-group in the incidence of UTIs (1.5 vs. $1.6 \%$ ) [25]. Genital infections reported as SAEs were rare in both treatment groups in DECLARE-TIMI 58 (two events in each group). Fournier's gangrene (necrotising fasciitis of the perineum) was reported in one dapagliflozin and five placebo recipients [25]; owing to the risk of this rare, but serious and potentially life-threatening event, patients should be advised to seek medical attention if they experience symptoms, and if Fournier's gangrene is suspected, dapagliflozin should be discontinued and prompt treatment should be instituted [7].

AEs of renal function occurred in $3 \%$ of dapagliflozin and $2 \%$ of placebo recipients in the 13 -study pooled analysis, occurring more frequently in patients with baseline eGFR $<60 \mathrm{~mL} /$ $\mathrm{min} / 1.73 \mathrm{~m}^{2}$ (vs. $\geq 60 \mathrm{~mL} / \mathrm{min} / 1.73 \mathrm{~m}^{2} ; 19$ vs. $1 \%$ in the dapagliflozin group) and those aged $\geq 65$ years (vs. $<65$ years; 8 vs. $2 \%$ in the dapagliflozin group) [38]. The most common renal AEs were decreased renal creatinine clearance (1.1 vs. $0.7 \%$ ) and renal impairment (0.8 vs. $0.5 \%)$, which were mostly transient, mild/moderate in severity and not accompanied with marked abnormalities of renal function. eGFR declined initially with dapagliflozin and returned towards baseline levels during treatement (mean change from baseline in the dapagliflozin group was -4.5 and $-1.5 \mathrm{~mL} / \mathrm{min} / 1.73 \mathrm{~m}^{2}$ at weeks 1 and 24 , respectively) [38]. In DECLARE-TIMI 58, the incidence of acute kidney injury was significantly lower with dapagliflozin than placebo (1.5 vs. $2.0 \% ; p=0.002)$ [25].

In the 13-study pooled analysis, volume depletionrelated AEs (hypotension, hypovolaemia and dehydration) were reported in $1.1 \%$ of dapagliflozin and $0.7 \%$ of placebo recipients, with half of the events in both groups occurring during the first 8 weeks of therapy (19 and $18 \%$ of AEs in the respective groups occurred within the first 2 weeks) [38]. Regardless of the treatment group, volume depletion-related AEs were more frequent in patients using loop diuretics than in those not using them (incidence 2.5-fold higher), and in patients with eGFR $<60 \mathrm{~mL} / \mathrm{min} / 1.73 \mathrm{~m}^{2}$ than in those with $\mathrm{eGFR} \geq 60 \mathrm{~mL} / \mathrm{min} / 1.73 \mathrm{~m}^{2}$ (incidence $\approx$ twofold higher). In 
the dapagliflozin group, patients aged $\geq 65$ years were also more likely (incidence $\approx$ twofold higher) to have volume depletion-related AEs than patients aged $<65$ years [38]. In DECLARE-TIMI 58, there was no significant difference between dapagliflozin and placebo recipients for symptoms of volume depletion (2.5 vs. 2.4\%) [25]. Dapagliflozin is not recommended for patients receiving loop diuretics [7].

Treatment with dapagliflozin was associated with small increases in parathyroid hormone, with larger increases seen in patients with higher baseline parathyroid levels [7, $14,39]$. No bone loss was observed with dapagliflozin during 2 years' therapy in patients with normal or mild renal impairment [7, 40]. Fractures were infrequent with dapagliflozin and placebo in the 13 -study pooled analysis ( $0.3 \mathrm{vs.}$ 0.7\%) [38]; in DECLARE-TIMI 58, there was no significant between-group difference in fracture rate (5.3 vs. 5.1\%) [25].

SGLT2 inhibitors, including dapagliflozin, have been associated with rare cases of diabetic ketoacidosis [DKA; hyperglycaemia ( $>250 \mathrm{mg} / \mathrm{dL}$ ), anion gap acidosis and increased plasma ketones], including life-threatening and fatal cases [7]. In a pooled analysis of 21 placebo-/active comparator-controlled trials of $\leq 208$ weeks' duration ( $n=5936$ in dapagliflozin and 3403 in control groups), one SAE of DKA (which may have occurred because of insulin dose reduction), two AEs of ketonuria and one AE of metabolic acidosis were reported with dapagliflozin versus no events in the control group (estimated incidence of DKA alone $0.02 \%$; $95 \%$ CI $0.004-0.059$ and of DKA/metabolic acidosis 0.03\%; 95\% CI 0.01-0.09) [38]. In DECLARETIMI 58, DKA occurred in significantly more dapagliflozin than placebo recipients $(0.3$ vs. $0.1 \% ; p=0.02)$ [25]. Prior to initiating dapagliflozin, factors in patients' history that may predispose them to ketoacidosis should be taken into consideration [7]. The risk of DKA should be considered if nonspecific symptoms (e.g. nausea, vomiting, anorexia) occur, and treatment should be discontinued if DKA is confirmed. Patients with euglycaemic DKA (DKA without hyperglycaemia) may need glucose in addition to standard treatment for DKA, and dapagliflozin should be discontinued if DKA occurs [7].

In a 21-study pooled analysis, although the incidence rate ratio (IRR) associated with dapagliflozin was above 1 for some tumours [bladder (IRR 5.2), breast (2.5), pancreatic (1.8)] and below 1 for other tumours [e.g. blood and lymphatic (0.4), renal tract (0.4)], the overall incidence rate of malignancies did not differ significantly between the dapagliflozin and control groups [1.5 vs. $1.5 \%$; IRR 1.03 ; $95 \%$ CI 0.7-1.5] [41]. In DECLARE-TIMI 58, bladder cancer occurred in fewer dapagliflozin than placebo recipients $(0.3$ vs. $0.5 \% ; p=0.02)$, and there was no between-group difference in the rate of breast cancer (0.4 vs. $0.4 \%$ ) [25].

Lower limb amputations were reported infrequently in the dapagliflozin and control groups (0.1 vs. $0.2 \%)$ in a 30 -study pooled analysis of placebo-/active comparator-controlled trials of $\geq 12$ weeks' duration ( $n=9195$ in dapagliflozin and 4629 in control groups) [38]. The time to amputation was similar in both groups and patients who had an amputation had a high prevalence of risk factors (e.g. neuropathy, CVD, dyslipidaemia and nephropathy) [38]. The rate of amputation did not differ significantly between dapagliflozin and placebo recipients in DECLARE-TIMI 58 (1.4 vs. 1.3\%) [25].

\subsection{Cardiovascular Safety}

A prespecified meta-analysis of $\mathrm{CV}$ events from 21 placebo-/ active comparator-controlled phase $2 b / 3$ clinical studies of $\leq$ 208 weeks' duration indicated that treatment with dapagliflozin was not associated with an increased $\mathrm{CV}$ risk in patients with $\mathrm{T} 2 \mathrm{D}$, and suggested a potential CV benefit with treatment, as evidenced by HRs of $<1$ for CV outcomes [42]. In the dapagliflozin and control groups, the event rate/100 patient-years (pt-y) of MACE plus unstable angina was 1.5 versus 2.2 in the overall population (HR $0.79 ; 95 \%$ CI $0.58-1.1$ ), 2.9 versus 3.8 in patients with CVD (HR 0.81; 95\% CI 0.56-1.16) and 4.2 versus 5.1 in elderly (aged $\geq 65$ years) patients with CVD risk (HR 0.82; 95\% CI 0.5-1.37). DECLARE-TIMI 58 confirmed the $\mathrm{CV}$ safety of dapagliflozin in patients with $\mathrm{T} 2 \mathrm{D}$ who had or were at risk of ASCVD, demonstrating noninferiority between dapagliflozin and placebo for the primary composite safety outcome of MACE $(p<0.001$ for noninferiority), and superiority for one of the two dual composite efficacy outcomes (CV death/HHF; Sect. 3.2) (Table 3) [25].

\section{Dosage and Administration}

In the EU, dapagliflozin is approved for use as monotherapy (in patients who are intolerant of metformin) and add-on combination therapy (with other glucose-lowering agents, including insulin) in patients with T2D when diet and exercise alone do not provide adequate glycaemic control [7]. The recommended dose of dapagliflozin is $10 \mathrm{mg}$ once daily administered orally, with or without food. When dapagliflozin is used in combination with insulin or insulin secretagogues (e.g. sulfonylureas), a lower dose of insulin or insulin secretagogues may be required because of an increased risk of hypoglycaemia. Dapagliflozin should not be initiated in patients with GFR $<60 \mathrm{~mL} /$ min and its use should be discontinued in patients with GFR persistently $<45 \mathrm{~mL} / \mathrm{min}$. No dosage adjustment is required based on renal function or in patients with mild or moderate hepatic impairment. In patients with severe hepatic impairment, dapagliflozin should be initiated at a dose of $5 \mathrm{mg} / \mathrm{day}$, and if well tolerated the dose should be increased to $10 \mathrm{mg} /$ day [7]. Local prescribing information should be consulted for further information, including dosage and administration details, contraindications, warnings and precautions. 


\section{Place of Dapagliflozin in the Management of T2D}

The aim of treatment in T2D is to prevent complications and optimize patient quality of life [43]. The 2018 American Diabetes Association (ADA) and European Association for the Study of Diabetes (EASD) consensus guidelines [43] and the 2019 ADA guidelines [44] recommend a patient-centred approach for the management of glycaemia and $\mathrm{CV}$ risk factors in T2D. Glycaemic targets should be individualized based on the risk of adverse events (e.g. hypoglycaemia and bodyweight gain), patient characteristics (e.g. comorbidity and patient frailty) and patient preference and goals [43]. Several classes of AHAs with different mechanisms of action are available for use in T2D. Metformin (unless contraindicated or not tolerated) and comprehensive lifestyle changes (including bodyweight management and physical activity) are first-line therapy. The choice of other AHAs should be individualized based on patient factors (including history of CVD, bodyweight, hypoglycaemic risk and CKD), the cost of treatment and patient preference [43]. CVD is the major cause of mortality in T2D, with MI and stroke accounting for $\approx 80 \%$ of all deaths [45]. Therefore, it is important that the AHA selected to improve glycaemic control in patients with T2D does not aggravate, and preferably improves, CV risk factors and reduces CV morbidity and mortality [45].

SGLT2 inhibitors are a relatively new class of oral AHAs that reduce plasma glucose levels by increasing urinary glucose excretion [46]. Because of their insulin-independent mechanism of action, SGLT2 inhibitors can be combined with other AHAs (including insulin) with minimal risk of hypoglycaemia [46]. Currently approved SGLT2 inhibitors in the EU include dapagliflozin, canagliflozin, empagliflozin and ertugliflozin, which are administered orally once daily. Dapagliflozin is a potent, highly selective SGLT2 inhibitor (Sect. 2) with proven efficacy and safety in patients with T2D (Sect. 3). In well-designed phase 3-4 clinical trials, dapagliflozin once daily as monotherapy and combination therapy with other AHAs, provided effective glycaemic control and reduced bodyweight and BP in a broad spectrum of T2D patients, including those with hypertension and/or CVD (Sect. 3). Evidence from real-world studies supported the efficacy of dapagliflozin in patients with T2D (Sect. 3.3).

Additionally, dapagliflozin was noninferior in terms of MACE and significantly lowered the rate of CV death and $\mathrm{HHF}$ relative to placebo in the large DECLARE-TIMI $58 \mathrm{CV}$ outcomes trial in patients at high risk for $\mathrm{CV}$ events, with the between-group difference largely attributed to a lower rate of HHF with dapagliflozin (Sect. 3.2). Dapagliflozin also reduced the likelihood of progression of renal disease, although statistical significance of these findings was not demonstrated because of hierarchical testing (Sect. 3.2). The CV and renal benefits with dapagliflozin were consistent across subgroups, suggesting treatment benefits across a broad patient population, regardless of history of ASCVD, HF or CKD at baseline (Sect. 3.2.1). Other subgroup analyses suggested that dapagliflozin reduced both MACE and CV death/HHF in high-risk patients with T2D and prior MI [29], and reduced CV death/ HHF to a greater extent in patients with HFrEF than in those without HFrEF (mainly because of a larger reduction in CV death in patients with HFrEF) [30] (Sect. 3.2.1). The ongoing phase 3 DAPA-HF (NCT03036124) trial in patients with confirmed HFrEF and the phase 3 DELIVER (NCT03619213) and phase 4 PRESERVED-HF (NCT03030235) trials in patients with preserved ejection fraction HF are further evaluating the effects of dapagliflozin in these patient subgroups, while the phase 3 DAPA-CKD (NCT03036150) trial in patients with CKD is assessing whether dapagliflozin delays the progression of kidney disease.

Dapagliflozin was generally well tolerated, with a low risk of hypoglycaemia and drug class-related AEs, including AEs of volume depletion, lower limb amputations, acute kidney injury and bladder cancer (Sect. 4). DKA (rare) and genital infections (common), also drug-class related, were reported more frequently with dapagliflozin than placebo; Fournier's gangrene was reported in one dapagliflozin and five placebo recipients in DECLARE-TIMI 58 (Sect. 4.1).

In addition to DECLARE-TIMI 58, CV and renal benefits with SGLT2 inhibitors were also seen in the EMPA-REG OUTCOME trial of empagliflozin [27] and the CANVAS Program for canagliflozin [47]. EMPA-REG OUTCOME exclusively enrolled patients with ASCVD, while DECLARE-TIMI 58 and the CANVAS Program had only $41 \%$ and $65 \%$ of patients with established ASCVD [25, 27, 47]. In all three CV outcomes trials, a more consistent and robust effect of SGLT2 inhibitors was seen for the prevention of HF and renal outcomes than in terms of atherosclerotic CV events [25]. This difference may be because of the mechanism of action of SGLT2 inhibitors on the kidney and other effects, such as natriuresis, reduction in BP and improvement in endothelial function [25]. Across the trials, SGLT2 inhibitors also appeared to moderately reduce the risk of MACE in patients with ASCVD, but not in patients with multiple risk factors [48]. However, in contrast to results from EMPA-REG OUTCOME, the rate of CV death and allcause death was not significantly reduced in DECLARE-TIMI 58 , which may be because of differences between the drugs or between the study designs [25].

Results from a recent meta-analysis of the three CV outcome trials of SGLT2 inhibitors were consistent with the findings from the individual trials, demonstrating robust benefits in terms of reducing HHF and progression of renal disease, and moderate benefits in terms of MACE, largely in patients with ASCVD [48]. Several mechanisms have been proposed to explain the CV benefits with SGLT2 inhibitors, including improvement in ventricular loading conditions, improvement in cardiac metabolism and bioenergetics, 
inhibition of myocardial $\mathrm{Na}^{+} / \mathrm{H}^{+}$exchange, reduction of necrosis and cardiac fibrosis, as well as alteration in adipokines, cytokine production and epicardial adipose tissue mass [49]

Among other AHAs that have been assessed in CV outcome trials, the GLP-1RAs liraglutide (LEADER) and semaglutide (SUSTAIN-6) significantly reduced the likelihood of MACE in patients with T2D, while exenatide (EXSCEL) and lixisenatide (ELIXA) did not demonstrate either a CV benefit or harm $[43,50]$. No significant effect of GLP-1RAs on HHF was observed in the CV outcome trials $[43,50]$. Results from a recent meta-analysis suggested that SGLT2 inhibitors reduced the risk of HHF to a greater extent than GLP-1RAs (SGLT2 inhibitors vs. GLP-1RAs: HR 0.71), while there was no significant difference between SGLT2 inhibitors and GLP-1RAs for the reduction in the risk of MACE (GLP-1RAs vs. SGLT2 inhibitors: HR 1.02) [51]. In CV outcome trials assessing DPP-4is, sitagliptin (TECOS), saxagliptin (SAVOR-TIMI 53) and alogliptin (EXAMINE) demonstrated CV safety, but no CV benefit, although saxagliptin was associated with a $27 \%$ greater $(p=0.007)$ risk of HHF than placebo [43, 50]. In terms of renal outcomes, unlike SGLT2 inhibitors that significantly reduced albuminuria and the decline in eGFR, GLP-1RAs were generally associated with significant reductions in albuminuria, but had no significant effect on eGFR, while the effect of DPP-4is on renal outcomes is unclear and needs further assessment [52].

CV benefits with SGLT2 inhibitors have also been seen in large real-world studies, including the observational CVDREAL study that enrolled $>300,000$ propensity score-matched T2D patients across six countries (USA, UK, Norway, Denmark, Sweden and Germany) [53]. Treatment with an SGLT2 inhibitor was associated with a lower risk of death (HR 0.49; $p<0.001$ ) and HHF (HR 0.61; $p<0.001$ ) [53], as well as a modestly lower risk of MI (HR $0.85 ; p=0.05$ ) and stroke (HR $0.83 ; p=0.02$ ) in patients newly initiated on an SGLT2 inhibitor (of the exposure time, 53, 42, and 5\% of patients received canagliflozin, dapagliflozin and empagliflozin, respectively) or another AHA ( $n=154,528$ patients/group). In a subgroup analysis, SGLT2 inhibitors reduced the risk of HF regardless of pre-existing CVD, a finding consistent with the results of clinical CV outcome trials [54]. Cardioprotective effects of SGLT2 inhibitors were also seen in the CVD-REAL Nordic (Denmark, Norway and Sweden; $n=40,908$ ) [55] and CVDREAL 2 (Asia Pacific, the Middle East and North American regions; $n=235,064$ ) [56] studies, which showed that initiation of an SGLT2 inhibitor was associated with a lower risk of $\mathrm{CV}$ events (including MACE and HHF) and all-cause death.

Given the CV benefits of SGLT2 inhibitors and GLP-1RAs, the recent $\mathrm{ADA} / \mathrm{EASD}$ consensus guidelines [43] and the ADA guidelines [44] recommend that history of CVD be considered very early during treatment selection for patients with T2D. In patients with established ASCVD who do not achieve $\mathrm{HbA}_{1 \mathrm{c}}$ target with metformin (or if metformin is not tolerated or contraindicated), the addition of an SGLT2 inhibitor or GLP-1RA with proven CVD benefit is recommended. In those with HF or CKD, the addition of an SGLT2 inhibitor with evidence of reducing $\mathrm{HF}$ and/or $\mathrm{CKD}$ progression is preferred, and if the SGLT2 inhibitor is not tolerated or contraindicated, the addition of a GLP-1RA with proven CKD benefit is recommended. If $\mathrm{HbA}_{1 \mathrm{c}}$ still remains above target and further intensification is required (or SGLT2 inhibitor or GLP-1RA is not tolerated), the guidelines recommend addition of the other drug class with proven CV benefit (GLP-1RA or SGLT2 inhibitor), a DPP$4 i$ (if not on GLP-1RA; not saxagliptin in the setting of HF), basal insulin, a thiazolidinedione (avoid in the setting of HF) or sulfonylurea. SGLT2 inhibitors are also recommended as second- and subsequent-line options for patients without history of ASCVD or CKD and with a compelling need to minimize hypoglycaemia or bodyweight [43, 44].

To conclude, oral dapagliflozin once daily improves glycaemic control, bodyweight and BP, and reduces the risk of $\mathrm{CV}$ death/HHF and possibly progression of renal disease, providing an important option for the management of a broad patient population, regardless of the history of CVD.

\section{Data Selection: 760 records identified \\ Duplicates removed \\ Excluded during initial screening (e.g. press releases; news reports; not relevant drug/indication; preclinical study; reviews; case reports; not randomized trial) \\ Excluded during writing (e.g. reviews; duplicate data; small patient number; nonrandomized/phase I/II trials) \\ Cited efficacy/tolerability articles 44 Cited articles not efficacy/tolerability 22 \\ Search Strategy: EMBASE, MEDLINE and PubMed from 2014 to present. Previous Adis Drug Evaluation published in 2014 was hand-searched for relevant data. Clinical trial registries/databases and websites were also searched for relevant data. Key words were dapagliflozin, Farxiga, Forxiga, BMS-512148, T2DM, type 2 diabete smellitus. Records were limited to those in English language. Searches last updated 10 June 2019}

Acknowledgements During the peer review process, the manufacturer of dapagliflozin was also offered an opportunity to review this article. Changes resulting from comments received were made on the basis of scientific and editorial merit

\section{Compliance with Ethical Standards}

Funding The preparation of this review was not supported by any external funding. 
Conflict of interest Sohita Dhillon is a salaried employee of Adis International Ltd/Springer Nature, is responsible for the article content and declares no relevant conflicts of interest.

Open Access This article is distributed under the terms of the Creative Commons Attribution-NonCommercial 4.0 International License (http://creativecommons.org/licenses/by-nc/4.0/), which permits any noncommercial use, duplication, adaptation, distribution and reproduction in any medium or format, as long as you give appropriate credit original author(s) and the source, provide a link to the Creative Commons licence and indicate if changes were made.

\section{References}

1. Hsia DS, Grove O, Cefalu WT. An update on sodium-glucose co-transporter-2 inhibitors for the treatment of diabetes mellitus. Curr Opin Endocrinol Diabetes Obes. 2017;24(1):73-9.

2. Wilding J, Fernando K, Milne N, et al. SGLT2 inhibitors in type 2 diabetes management: key evidence and implications for clinical practice. Diabetes Ther. 2018;9(5):1757-73.

3. Scheen AJ. Pharmacodynamics, efficacy and safety of sodiumglucose co-transporter type 2 (SGLT2) inhibitors for the treatment of type 2 diabetes mellitus. Drugs. 2015;75(1):33-59.

4. Plosker GL. Dapagliflozin: a review of its use in type 2 diabetes mellitus. Drugs. 2012;72(17):2289-312.

5. Plosker GL. Dapagliflozin: a review of its use in patients with type 2 diabetes. Drugs. 2014;74(18):2191-209.

6. Meng W, Ellsworth BA, Nirschl AA, et al. Discovery of dapagliflozin: a potent, selective renal sodium-dependent glucose cotransporter 2 (SGLT2) inhibitor for the treatment of type 2 diabetes. J Med Chem. 2008;51(5):1145-9.

7. AstraZeneca. Forxiga (dapagliflozin): summary of product characteristics. 2019. https://www.ema.europa.eu/en. Accessed 4 Jun 2019.

8. Komoroski B, Vachharajani N, Feng Y, et al. Dapagliflozin, a novel, selective SGLT2 inhibitor, improved glycemic control over 2 weeks in patients with type 2 diabetes mellitus. Clin Pharmacol Ther. 2009;85(5):513-9.

9. Bailey CJ, Gross JL, Pieters A, et al. Effect of dapagliflozin in patients with type 2 diabetes who have inadequate glycaemic control with metformin: a randomised, double-blind, placebocontrolled trial. Lancet. 2010;375(9733):2223-33.

10. Heerspink HJL, Kurlyandskaya R, Xu J, et al. Differential effects of dapagliflozin on cardiovascular risk factors at varying degrees of renal function. Diabetes. 2016;65(Suppl 1):A286.

11. Scheen AJ, Paquot N. Metabolic effects of SGLT-2 inhibitors beyond increased glucosuria: a review of the clinical evidence. Diabetes Metab. 2014;40(6 Suppl 1):S4-11.

12. Skolnik N, Bonnes H, Yeh H, et al. Dapagliflozin in the treatment of patients with type 2 diabetes presenting with high baseline A1C. Postgrad Med. 2016;128(4):356-63.

13. Fioretto P, Mansfield TA, Ptaszynska A, et al. Long-term safety of dapagliflozin in older patients with type 2 diabetes mellitus: a pooled analysis of phase IIb/III studies. Drugs Aging. 2016;33(7):511-22.

14. Kohan DE, Fioretto P, Tang W, et al. Long-term study of patients with type 2 diabetes and moderate renal impairment shows that dapagliflozin reduces weight and blood pressure but does not improve glycemic control. Kidney Int. 2014;85(4):962-71.

15. Weber MA, Mansfield TA, Alessi F, et al. Effects of dapagliflozin on blood pressure in hypertensive diabetic patients on reninangiotensin system blockade. Blood Press. 2016;25(2):93-103.

16. Weber MA, Mansfield TA, Cain VA, et al. Blood pressure and glycaemic effects of dapagliflozin versus placebo in patients with type 2 diabetes on combination antihypertensive therapy: a randomised, double-blind, placebo-controlled, phase 3 study. Lancet Diabetes Endocrinol. 2016;4(3):211-20.

17. Leiter LA, Cefalu WT, de Bruin TW, et al. Dapagliflozin added to usual care in individuals with type 2 diabetes mellitus with preexisting cardiovascular disease: a 24 -week, multicenter, randomized, double-blind, placebo-controlled study with a 28-week extension. J Am Geriatr Soc. 2014;62(7):1252-62.

18. Cefalu WT, Leiter LA, de Bruin TWA, et al. Dapagliflozin's effects on glycemia and cardiovascular risk factors in high-risk patients with type 2 diabetes: a 24-week, multicenter, randomized, double-blind, placebo-controlled study with a 28-week extension. Diabetes Care. 2015;38(7):1218-27.

19. Bolinder J, Ljunggren O, Kullberg J, et al. Effects of dapagliflozin on body weight, total fat mass, and regional adipose tissue distribution in patients with type 2 diabetes mellitus with inadequate glycemic control on metformin. J Clin Endocrinol Metab. 2012;97(3):1020-31.

20. Bolinder J, Ljunggren O, Johansson L, et al. Dapagliflozin maintains glycaemic control while reducing weight and body fat mass over 2 years in patients with type 2 diabetes mellitus inadequately controlled on metformin. Diabetes Obes Metab. 2014;16(2):159-69.

21. Leiter LA, Cefalu WT, de Bruin TW, et al. Long-term maintenance of efficacy of dapagliflozin in patients with type 2 diabetes mellitus and cardiovascular disease. Diabetes Obes Metab. 2016;18(8):766-74.

22. Kosiborod M, Gause-Nilsson I, Xu J, et al. Efficacy and safety of dapagliflozin in patients with type 2 diabetes and concomitant heart failure. J Diabetes Complicat. 2017;31(7):1215-21.

23. Fioretto P, Del Prato S, Buse JB, et al. Efficacy and safety of dapagliflozin in patients with type 2 diabetes and moderate renal impairment (chronic kidney disease stage 3A): the DERIVE study. Diabetes Obes Metab. 2018;20(11):2532-40.

24. Scott R, Morgan J, Zimmer Z, et al. A randomized clinical trial of the efficacy and safety of sitagliptin compared with dapagliflozin in patients with type 2 diabetes mellitus and mild renal insufficiency: the CompoSIT-R study. Diabetes Obes Metab. 2018;20(12):2876-84.

25. Wiviott SD, Raz I, Bonaca MP, et al. Dapagliflozin and cardiovascular outcomes in type 2 diabetes. $\mathrm{N}$ Engl $\mathrm{J}$ Med. 2018;380(4):347-357.

26. Raz I, Mosenzon O, Bonaca MP, et al. DECLARE-TIMI 58: participants' baseline characteristics. Diabetes Obes Metab. 2018;20(5):1102-10.

27. Zinman B, Wanner C, Lachin JM, et al. Empagliflozin, cardiovascular outcomes, and mortality in type 2 diabetes. N Engl J Med. 2015;373(22):2117-28.

28. Mosenzon O, Wiviott SD, Cahn A, et al. Effects of dapagliflozin on development and progression of kidney disease in patients with type 2 diabetes: an analysis from the DECLARE-TIMI 58 randomised trial. Lancet Diabetes Endocrinol. 2019. doi: https:// doi.org/10.1016/S2213-8587(19)30180-9.

29. Furtado RHM, Bonaca MP, Raz I, et al. Dapagliflozin and cardiovascular outcomes in patients with type 2 diabetes and prior myocardial infarction: a sub-analysis from DECLARE TIMI-58 trial. Circulation. 2019;139(22):2516-27.

30. Kato ET, Silverman MG, Mosenzon O, et al. Effect of dapagliflozin on heart failure and mortality in type 2 diabetes mellitus. Circulation. 2019;139(22):2528-36.

31. Wilding J, Bailey C, Rigney U, et al. Dapagliflozin therapy for type 2 diabetes in primary care: effects on $\mathrm{HbAlc}$, weight and blood pressure over two years follow-up [abstract no. P508]. Diabet Med. 2017;34(Suppl 1):189.

32. Huang H, Bell K, Gani R, et al. A retrospective real-world study of dapagliflozin vs. other oral antidiabetic therapies added to 
metformin in patients with type 2 diabetes (T2D) [abstract no. 1233-P]. Diabetes. 2017;66(Suppl 1):A330.

33. Han E, Kim A, Lee SJ, et al. Characteristics of dapagliflozin responders: a longitudinal, prospective, nationwide dapagliflozin surveillance study in Korea. Diabetes Ther. 2018;9(4):1689-701.

34. Viswanathan V, Singh KP. Usage of dapagliflozin, a sodium glucose co-transporter inhibitor, in the management of type-2 diabetes mellitus: a real world evidence study in Indian patients (FOREFRONT) [abstract no. PP50]. Int J Diabetes Dev Ctries. 2018;38(Suppl 2):S155.

35. Brown RE, Abitbol A, Aronson R. Real-world health outcomes of dapagliflozin vs. a DPP-4 inhibitor or a sulfonylurea in type 2 diabetes. Diabetes. 2017;66(Suppl. 1):A347.

36. Fadini GP, Zatti G, Baldi I, et al. Use and effectiveness of dapagliflozin in routine clinical practice: an Italian multicentre retrospective study. Diabetes Obes Metab. 2018;20(7):1781-6.

37. Persson F, Nystrom T, Jorgensen ME, et al. Dapagliflozin is associated with lower risk of cardiovascular events and all-cause mortality in people with type 2 diabetes (CVD-REAL Nordic) when compared with dipeptidyl peptidase-4 inhibitor therapy: a multinational observational study. Diabetes Obes Metab. 2018;20(2):344-51.

38. Jabbour S, Seufert J, Scheen A, et al. Dapagliflozin in patients with type 2 diabetes mellitus: a pooled analysis of safety data from phase IIb/III clinical trials. Diabetes Obes Metab. 2018;20(3):620-8.

39. Bailey CJ, Morales Villegas EC, Woo V, et al. Efficacy and safety of dapagliflozin monotherapy in people with type 2 diabetes: a randomized double-blind placebo-controlled 102-week trial. Diabet Med. 2015;32(4):531-41.

40. Ljunggren O, Bolinder J, Johansson L, et al. Dapagliflozin has no effect on markers of bone formation and resorption or bone mineral density in patients with inadequately controlled type 2 diabetes mellitus on metformin. Diabetes Obes Metab. 2012;14(11):990-9.

41. Ptaszynska A, Cohen SM, Messing EM, et al. Assessing bladder cancer risk in type 2 diabetes clinical trials: the dapagliflozin drug development program as a 'case study'. Diabetes Ther. 2015;6(3):357-75.

42. Sonesson C, Johansson PA, Johnsson E, et al. Cardiovascular effects of dapagliflozin in patients with type 2 diabetes and different risk categories: a meta-analysis. Cardiovasc Diabetol. 2016;15:37.

43. Davies MJ, D'Alessio DA, Fradkin J, et al. Management of hyperglycemia in type 2 diabetes, 2018: a consensus report by the American Diabetes Association (ADA) and the European Association for the Study of Diabetes (EASD). Diabetes Care. 2018;41(12):2669-701.

44. American Diabetes Association. 9. Pharmacologic approaches to glycemic treatment: standards of medical care in diabetes-2019 [web annotation]. Diabetes Care. 2019;42(Suppl 1):S90-102.

45. Ferrannini E, DeFronzo RA. Impact of glucose-lowering drugs on cardiovascular disease in type 2 diabetes. Eur Heart J. 2015;36(34):2288-96.

46. Lupsa BC, Inzucchi SE. Use of SGLT2 inhibitors in type 2 diabetes: weighing the risks and benefits. Diabetologia. 2018;61(10):2118-25.

47. Neal B, Perkovic V, Mahaffey KW, et al. Canagliflozin and cardiovascular and renal events in type 2 diabetes. $N$ Engl J Med. 2017;377(7):644-57.

48. Zelniker TA, Wiviott SD, Raz I, et al. SGLT2 inhibitors for primary and secondary prevention of cardiovascular and renal outcomes in type 2 diabetes: a systematic review and meta-analysis of cardiovascular outcome trials. Lancet. 2019;393(10166):31-9.

49. Verma S, McMurray JJV. SGLT2 inhibitors and mechanisms of cardiovascular benefit: a state-of-the-art review. Diabetologia. 2018;61(10):2108-17.

50. Bailey CJ, Marx N. Cardiovascular protection in type 2 diabetes: insights from recent outcome trials. Diabetes Obes Metab. 2019;21(1):3-14.

51. Hussein H, Zaccardi F, Khunti K, et al. Cardiovascular efficacy and safety of sodium-glucose co-transporter- 2 inhibitors and glucagon-like peptide-1 receptor agonists: a systematic review and network meta-analysis. Diabet Med. 2019;36(4):444-52.

52. Scheen AJ. Effects of glucose-lowering agents on surrogate endpoints and hard clinical renal outcomes in patients with type 2 diabetes. Diabetes Metab. 2019;45(2):110-21.

53. Kosiborod M, Cavender MA, Fu AZ, et al. Lower risk of heart failure and death in patients initiated on sodium-glucose cotransporter-2 inhibitors versus other glucose-lowering drugs: the CVD-REAL study (comparative effectiveness of cardiovascular outcomes in new users of sodium-glucose cotransporter- 2 inhibitors). Circulation. 2017;136(3):249-59.

54. Cavender MA, Norhammar A, Birkeland KI, et al. SGLT-2 inhibitors and cardiovascular risk: an analysis of CVD-REAL. J Am Coll Cardiol. 2018;71(22):2497-506.

55. Birkeland KI, Jorgensen ME, Carstensen B, et al. Cardiovascular mortality and morbidity in patients with type 2 diabetes following initiation of sodium-glucose co-transporter-2 inhibitors versus other glucose-lowering drugs (CVD-REAL Nordic): a multinational observational analysis. Lancet Diabetes Endocrinol. 2017;5(9):709-17.

56. Kosiborod M, Lam CSP, Kohsaka S, et al. Cardiovascular events associated with SGLT-2 inhibitors versus other glucoselowering drugs: the CVD-REAL 2 study. J Am Coll Cardiol. 2018;71(23):2628-39.

57. Matthaei S, Bowering K, Rohwedder K, et al. Durability and tolerability of dapagliflozin over 52 weeks as add-on to metformin and sulphonylurea in type 2 diabetes. Diabetes Obes Metab. 2015;17(11):1075-84.

58. Mathieu C, Herrera Marmolejo M, Gonzalez Gonzalez JG, et al. Efficacy and safety of triple therapy with dapagliflozin add-on to saxagliptin plus metformin over 52 weeks in patients with type 2 diabetes. Diabetes Obes Metab. 2016;18(11):1134-7.

59. Vilsboll T, Ekholm E, Johnsson E, et al. Efficacy of dapagliflozin plus saxagliptin vs insulin glargine at 52 weeks in patients with type 2 diabetes inadequately controlled by metformin with or without sulfonylurea [abstract no. 775]. Diabetologia. 2018;61(Suppl 1):S380.

60. Jabbour S, Guja C, Hardy E, et al. DURATION-8 randomized controlled trial 104-week results-once-weekly exenatide (EXQW) plus once-daily dapagliflozin (DAPA) vs. Exqw or DAPA alone [abstract no. 104-LB]. Diabetes. 2018;67(Suppl 1):LB29-30.

61. Frias JP, Guja C, Hardy E, et al. Exenatide once weekly plus dapagliflozin once daily versus exenatide or dapagliflozin alone in patients with type 2 diabetes inadequately controlled with metformin monotherapy (DURATION-8): a 28 week, multicentre, double-blind, phase 3, randomised controlled trial. Lancet Diabetes Endocrinol. 2016;4(12):1004-16.

62. Matthaei S, Bowering K, Rohwedder K, et al. Dapagliflozin improves glycemic control and reduces body weight as add-on therapy to metformin plus sulfonylurea: a 24 -week randomized, double-blind clinical trial. Diabetes Care. 2015;38(3):365-72.

63. Mathieu C, Ranetti AE, Li D, et al. Randomized, double-blind, phase 3 trial of triple therapy with dapagliflozin add-on to saxagliptin plus metformin in type 2 diabetes. Diabetes Care. 2015;38(11):2009-17.

64. Müller-Wieland D, Kellerer M, Cypryk K, et al. Efficacy and safety of dapagliflozin or dapagliflozin plus saxagliptin versus glimepiride as add-on to metformin in patients with type 2 diabetes. Diabetes Obes Metab. 2018;20(11):2598-607.

65. Handelsman Y, Mathieu C, Del Prato S, et al. Sustained 52-week efficacy and safety of triple therapy with dapagliflozin plus saxagliptin versus dual therapy with sitagliptin added to metformin in patients with uncontrolled type 2 diabetes. Diabetes Obes Metab. 2018. https://doi.org/10.1111/dom.13594.

66. Vilsboll T, Ekholm E, Johnsson EK, et al. Dapagliflozin plus saxagliptin shows noninferior $\mathrm{A} 1 \mathrm{C}$ reduction vs. insulin glargine in patients with type 2 diabetes inadequately controlled by metformin with or without sulfonylurea. Diabetes. 2018;67(Suppl 1):A70. 